\title{
Los periodistas españoles ante el tratamiento informativo de los grupos minoritarios y desfavorecidos'
}

\author{
Begoña ZaLBIDEA BENGOA \\ Universidad del País Vasco - Euskal Herriko Unibertsitatea (UPV/EHU) \\ bego.zalbidea@ehu.es \\ Juan Carlos PÉREZ Fuentes \\ Universidad del País Vasco - Euskal Herriko Unibertsitatea (UPV/EHU) \\ carlos.perez@ehu.es \\ Santiago URRUTIA IZAGUIRRE \\ Universidad del País Vasco - Euskal Herriko Unibertsitatea (UPV/EHU) \\ santi.urrutia@ehu.es \\ Susana LÓPEZ PÉREZ \\ Universidad del País Vasco - Euskal Herriko Unibertsitatea (UPV/EHU) \\ susana.lopezp@ehu.es
}

Recibido: 9/03/2011

Aceptado: 26/10/2011

\begin{abstract}
Resumen
La mayoría de los códigos deontológicos generalistas europeos recoge el deber profesional de los periodistas de no discriminar por razones de sexo, de etnia (o de raza) y de religión. Algunos textos van más allá y hablan del origen nacional, cultural o social, del idioma o de la ideología. El estudio se centra en el grado de asunción de dichas pautas en la praxis profesional de los periodistas de cuatro comunidades autónomas que representan el 52\% de toda la población del Estado Español: Euskadi, Andalucía, Cataluña y Madrid. Partiendo de la base de que su inobservancia afecta, sobre todo, a los colectivos desfavorecidos -tales como inmigrantes, mujeres, homosexuales, discapacitados, ancianos, etc.-, a los informadores se les plantea la cuestión de si a la hora de presentar la noticia es admisible algún tipo de discriminación 'positiva'.
\end{abstract}

Palabras clave: ética, deontología, periodistas, periodismo, discriminación, grupos desfavorecidos.

\section{How Spanish Journalists consider minorities and vulnerable groups should be treated by media}

\begin{abstract}
The majority of the general European codes include among their principles that professionals have a duty not to discriminate for reasons of sex, of etnia (or of race) and of religion. Some texts go beyond and speak about the national, cultural or social origin, language or ideology. This study focuses on how much are assumed these guidelines in the professional activity of journalists from four regions (autonomous communities) that add up to more than half of population of Spanish state (52\%): Andalusia, Catalonia, Madrid and Basque Country. The discrimination affects often to groups that are socially or economically deprived or marginalized -as immigrants, women, homosexuals, disabled and elderly peopleand, besides the usual questions that arise in this area, news people are faced with the issue whether is it admissible to act with some kind of 'positive discrimination'.
\end{abstract}

Keywords: Ethics, deontology, journalists, journalism, discrimination, deprived groups.

${ }^{1}$ Los datos que se recogen en este trabajo son resultado de dos proyectos de investigación subvencionados respectivamente por el MEC (código SEJ2006-05631-C05/ de 1-10-2006 a 303-2010) y el MICINN (código CSO2009-1380/ de 2-1-2010 a 31-12-2012) 


\section{Referencia normalizada}

ZALBIDEA BENGOA, Begoña; PÉREZ FUENTES, Juan Carlos; URRUTIA IZAGUIRRE, Santiago; y LÓPEZ PÉREZ, Susana (2011): "Los periodistas españoles ante el tratamiento informativo de los grupos minoritarios y desfavorecidos". Estudios sobre el mensaje periodístico. Vol. 17, núm. 2, págs.: 457476. Madrid, Servicio de Publicaciones de la Universidad Complutense.

Sumario: 1. Introducción . 2 Fuentes y metodología. 3. Los códigos deontológicos de los 27 estados de la UE. 4. La opinión de los periodistas. 5. Análisis por colectivos. 5.1. Mujeres. 5.2. Inmigrantes. 5.3. Gitanos. 5.4. Discapacitados. 5.5. Homosexuales. 5.6. Ancianos. 5.7. Personas con enfermedad mental. 6. Conclusiones. 7. Referencias bibliográficas. 8. Códigos deontológicos consultados.

\section{Introducción}

Es evidente que el poder que tienen los medios de comunicación nace de su capacidad para construir la realidad, de tal manera que los hechos y los procesos sociales objetivos son experimentados por los individuos a través de la narración e interpretación que de ellos hacen los medios. Como señala Gaye Tuchman (1983: 10), la noticia no es un espejo, sino una ventana al mundo a través de la cual "los ciudadanos aprenden sobre sí mismos y sobre otros, sobre las instituciones, los líderes y los estilos de vida y sobre otras naciones y sus gentes. La noticia tiende a decirnos qué queremos saber, qué necesitamos saber y qué deberíamos saber". Podemos afirmar, por tanto, que los medios de comunicación desempeñan un papel central en el enmarque interpretativo (framing) de la realidad.

La toma de conciencia del poder de la prensa como conformadora de la opinión pública, y la consiguiente alerta acerca de la capacidad de ésta para provocar daños en la sociedad, dio lugar a la Teoría de la Responsabilidad Social de los Medios y, por extensión, a la reflexión de los propios periodistas acerca de su responsabilidad en tanto que intermediarios entre el público y la actualidad. Es obvio que esta percepción de los medios de comunicación surge de una definición del periodismo que va más allá de su simple consideración como producto, para pasar a ser entendido como un bien social (UNESCO, 1983) ${ }^{2}$. Sin embargo, como señala el Consell de l'Audiovisual de Catalunya (2001), en Recomendaciones sobre el tratamiento informativo de las tragedias personales (Introducción), "muchas veces los profesionales no disponen de suficientes referentes que les facilite adoptar una actitud crítica y rigurosamente consciente del alcance y repercusión de su actividad, más lejos del propio genio, su buen sentido o la experiencia acumulada". De ahí que una de las consecuencias de la asunción de la Teoría de la Responsabilidad fuera la redacción de múltiples códigos éticos y deontológicos de carácter internacional, nacional, regional, sectorial e interno de los propios medios, cuya mera existencia supone el reconocimiento de que el ejercicio de esta profesión conlleva una serie de valores y obligaciones morales que deben acompañar y guiar su desempeño (AZNAR, 1997: 128).

${ }^{2}$ Principios internacionales de ética profesional del periodismo. Aprobado en la cuarta reunión consultiva de periodistas auspiciada por la UNESCO el 20 de noviembre de 1983. Éste y otros códigos pueden consultarse en PÉREZ FUENTES, 2004) 


\section{Fuentes y metodología}

El presente trabajo persigue dos objetivos: conocer lo que estipulan los principales códigos deontológicos de los periodistas de los 27 países de la Unión Europea, así como la opinión de los profesionales de los medios de comunicación que ejercen en Andalucía, Cataluña, Madrid y País Vasco, comunidades que en su conjunto representan el $52 \%$ de la población total censada del Estado. Para el primero de los objetivos se ha procedido a extraer la parte de los textos que hacen referencia a las siguientes categorías: sexo, cultura, religión, orientación sexual, lengua, opiniones, raza, origen nacional, origen social, enfermos y discapacitados, mayores, edad y color. En lo concerniente a la opinión de los informadores, se ha seguido la doble metodología cualitativa y cuantitativa. En la primera se han realizado 104 entrevistas en profundidad a profesionales de todo tipo de medios (prensa escrita, televisión, radio, Internet), de ambos sexos, de todas las franjas de edad y de todas las categorías profesionales. En lo referente al análisis cuantitativo, se han realizado más de dos mil encuestas entre las distintas zonas geográficas. La pregunta de referencia que se les ha planteado ha sido la siguiente: "Respecto a los siguientes colectivos, ¿en qué grado es partidario de que reciban un tratamiento informativo especial a causa de su situación desfavorecida?"

\section{Los códigos deontológicos de los 27 estados de la UE}

Fenómenos nuevos, mentalidades diferentes, sensibilidades recientes y procesos sociales de transformación obligan a una continua reflexión acerca del papel de los medios en la representación de las nuevas realidades. Al ser la sociedad más compleja crecen las dificultades para que la conciencia moral de los individuos pueda llegar por sí sola a determinar las normas que deben guiar la actividad de los periodistas (AZNAR, 1997: p. 128). Por ello, son necesarios los códigos deontológicos e imprescindible su revisión periódica. Los propios profesionales son conscientes de esta necesidad. Tal y como recoge el Consejo de Prensa de Irlanda y del Defensor del Pueblo (2008) en el Código de Buenas Prácticas para la Prensa y las Publicaciones Periódicas, "el Código de Buenas Prácticas se empleará para los trabajos en curso y se espera que siga evolucionando a la luz de la experiencia y en respuesta a los cambios en la opinión pública y las percepciones".

De entre los fenómenos a los que se enfrentan los informadores, y que han exigido una reflexión profunda, se encuentran todos aquellos asuntos que pueden generar algún tipo de discriminación. Es obvio que en las sociedades democráticas del mundo occidental la sensibilidad hacia los grupos desfavorecidos ha ido arraigando en los últimos años hasta llegar a convertirse en un valor asumido socialmente. Por tanto, Gobiernos, instituciones y movimientos sociales han iniciado la senda de aplicar políticas para evitar las actitudes y conductas discriminatorias y proteger a los grupos más débiles. Mujeres, menores, mayores, enfermos y grupos minoritarios (étnicos, religiosos y culturales) son los colectivos considerados especialmente vulnerables. Y los medios no son insensibles a esta preocupación, una preocupación que ha encontrado su lógica plasmación en los códigos éticos, en los cuales se expresa el deber profesional de no discriminar. 
El marco de referencia con que cuentan los periodistas para plasmar este deber se halla en los códigos internacionales. Así, bajo los auspicios de la UNESCO se aprobaron en 1983 unos 'Principios Internacionales' que afirman que el verdadero periodista "respeta el carácter distintivo, el valor y dignidad de cada cultura". Tres años más tarde la Federación Internacional de Periodistas recoge en el art. 7 de su Declaración de principios sobre la conducta de los periodistas (1986) la necesidad de impedir "los riesgos de una discriminación", haciendo todo lo posible para "evitar que se facilite tal discriminación, fundamentada especialmente en la raza, el sexo, la moral sexual, la lengua, la religión, las opiniones políticas y demás, así como el origen nacional o social". También el Consejo de Europa, en la 'Resolución 1003', recoge las obligaciones morales de los medios de comunicación en este terreno, pero va más allá al exigir que no utilicen el lenguaje del odio y del enfrentamiento ${ }^{3}$.

Esta sensibilidad hacia los grupos más desfavorecidos ha llevado a que la práctica totalidad de los códigos generalistas de los 27 Estados de la Unión Europea recojan entre sus indicaciones la del deber profesional de no discriminar, al igual que lo hacen el 'Código Deontológico de la Profesión Periodística' de la Federación de Asociaciones de Periodistas de España (FAPE) y la 'Declaración de Principios de la Profesión Periodística' del Colegio de Periodistas de Cataluña. Únicamente los de Francia y Eslovaquia no incluyen ninguna mención, mientras que en Holanda se rigen expresamente por el código de la Federación Internacional de Periodistas.

Aunque la mayoría hace expresa referencia a la prohibición de la discriminación en la información por razones de sexo, de etnia (o de raza) y de religión, algunos textos van más allá y hablan del origen nacional, cultural o social, del idioma o de la ideología de los protagonistas de las noticias, como factores que deben asimismo ser tenidos en cuenta. En los códigos europeos se deduce que apareciendo la raza en el $90 \%$ de los documentos, la religión (75\%), el origen nacional (58\%) y el color (30\%) aspectos a proteger, es el cuidado en el tratamiento informativo de los grupos inmigrantes una preocupación fundamental. Las mujeres (70\%) y los mayores (41\%) también son objeto de protección.

La realidad de la inmigración en Europa, con 19,5 millones de extranjeros procedentes de otros continentes en $2008^{4}$, corre paralela al riesgo de la xenofobia. De ahí la preocupación de los periodistas por el tratamiento informativo de los inmigrantes y sus descendientes. Si englobamos los términos de raza, origen étnico, color y origen

${ }^{3}$ La 'Resolución 1003 sobre la Ética del Periodismo' fue aprobada por Asamblea Parlamentaria del Consejo de Europa en 1993. En el apartado titulado 'Situaciones de conflicto y casos de protección especial', dice: "En la sociedad se dan a veces situaciones de tensión y de conflictos nacidos bajo la presión de factores como el terrorismo, la discriminación de las minorías, la xenofobia o la guerra. En estas circunstancias los medios de comunicación tienen la obligación moral de defender los valores de la democracia, el respeto de la dignidad humana, las solución de los problemas a través de métodos pacíficos y de tolerancia, y en consecuencia oponerse a la violencia y al lenguaje del odio o del enfrentamiento, rechazando toda discriminación por razón de cultura, sexo o religión" (art. 33).

${ }^{4}$ De ellos, 4,7 millones eran africanos, 3,7 millones, asiáticos y 3,2 millones procedían de América. Fuente: Eurostat Newsrelease 184/2009: Population of foreign citizen in EU27 in 2008. 
nacional bajo el paraguas de una misma realidad, todos los estados, salvo Malta, contemplan la no discriminación por este aspecto; aunque muchos se limitan a señalar que "no se discriminará por razón de raza, color u origen étnico", otros códigos son más concretos, como el de Alemania, que expresamente dicta la siguiente norma: "Al informar sobre hechos delictivos sólo se mencionará la pertenencia de los sospechosos o autores a una minoría religiosa, étnica o a otra cualquiera si ello es imprescindible para la mejor comprensión del contenido de la noticia. Debe considerarse que la mención de ciertos detalles puede avivar prejuicios con respecto a grupos que necesitan protección" (Normas Fundamentales del Periodismo, aprobadas por el Consejo Alemán de Prensa en colaboración con las Asociaciones de Prensa en 2006 (art. 12.1). Indicaciones similares se observan en otros códigos, como los de Bulgaria, Dinamarca, Lituania y Luxemburgo. Este último advierte que "hay formas de comunicación que sin incitar directamente a la discriminación o el odio pueden favorecer un ambiente que puede crear en el público sentimientos negativos hacia una comunidad", y aclara lo dicho con un ejemplo: "la práctica habitual que indica el color de la piel de una persona entrevistada cada vez que él o ella no es blanca" (Código deontológico del Consejo de Prensa de Luxemburgo, aprobado en 2004).

Una de las consecuencias de la llegada de inmigrantes a Europa es la diversidad cultural y religiosa. De entre los distintos credos que se practican en el continente, el islam ocupa el segundo lugar en número de seguidores y su importancia porcentual sigue creciendo. En Europa occidental viven al menos 18 millones de personas que pueden considerarse culturalmente musulmanas (ALLIVERI, 2010: 3). No es de extrañar, por tanto, que la mayoría de los códigos deontológicos europeos incidan en el respeto a las diversas creencias religiosas. Resulta curioso que esa consideración a las diferentes culturas haya tenido escasa cabida en estos textos orientadores, ya que sólo Bélgica, Polonia y Luxemburgo lo recogen expresamente, mientras que Hungría lo incluye refiriéndose a las distintas formas de vida.

Siguiendo los pasos dados por las administraciones públicas en la búsqueda de una igualdad efectiva entre hombres y mujeres, los periodistas se hacen eco de la importancia de no discriminar por razones de sexo. La consecución de la igualdad real, más allá de la promulgación de normas legales, camina de la mano de una conciencia social, y es ahí donde los medios de comunicación juegan un importante papel. Por ello, el $70 \%$ de los códigos consultados hace referencia expresa a esta cuestión. No existe la misma sensibilidad hacia la orientación sexual de los individuos, porque sólo ocho códigos se refieren a ella como factor de no discriminación.

Con una esperanza de vida de 75,6 años para los hombres y de 81,8 años para las mujeres, la población que supera los 65 años en Europa alcanzaba los 77 millones de personas en 2005 (Comisión Europea, 2007: Europe's demographic future: facts and figures). A pesar de ello, sólo cuatro de los códigos generalistas recogen la necesidad de proteger a los mayores, aunque otros seis hablan de no discriminar por razones de edad. Más preocupación se muestra hacia los enfermos y discapacitados, un grupo que es expresamente mencionado en trece de los códigos.

Finalmente, hay otros factores que son nombrados de forma dispar: en siete de los textos se señala que no se debe discriminar por el origen social del individuo; en el de 
Irlanda, por el estado civil; en Eslovenia, por el aspecto físico; en otros diez textos, por ideología u opiniones políticas; y en cuatro de ellos (Hungría, Bélgica, Chipre y Luxemburgo) se dice que no debe hacerse por razones de lengua. Llama la atención que en países como Irlanda o España, donde existe una realidad lingüística variada, no se haga mención de este factor.

\section{La opinión de los periodistas}

Los periodistas, que tienen en su haber la responsabilidad de transmitir la información de forma veraz, con rigor, imparcialidad y de manera equilibrada, parecen estar dispuestos a hacer concesiones a ciertos colectivos o, al menos, a los que en este trabajo se han agrupado bajo el denominador común de 'grupos desfavorecidos'. Esa cesión, empero, se presenta muy tasada y ante colectivos con escasa presencia en los espacios que los mass media reservan a la divulgación de cuantos hechos o acontecimientos acaecen a diario. Es, por tanto, una actitud permisiva con escasa o nula incidencia en la praxis de los articulistas.

Una primera aproximación a esa realidad aparece en el cuadro de respuestas dadas por los informadores de la Comunidad Autónoma Vasca. A quienes han participado en el trabajo de campo en Euskadi, como en otras comunidades, se les ha pedido la opinión que les merece otorgar un tratamiento informativo especial a los 'grupos desfavorecidos'. Así, y como puede apreciarse en la gráfica 1, sólo las personas discapacitadas, las que padecen alguna enfermedad mental y los ancianos merecerían un tratamiento informativo especial. En estos Gráfica 1.- Grado de apoyo de los periodistas a los grupos desfavorecidos

\section{Euskadi}

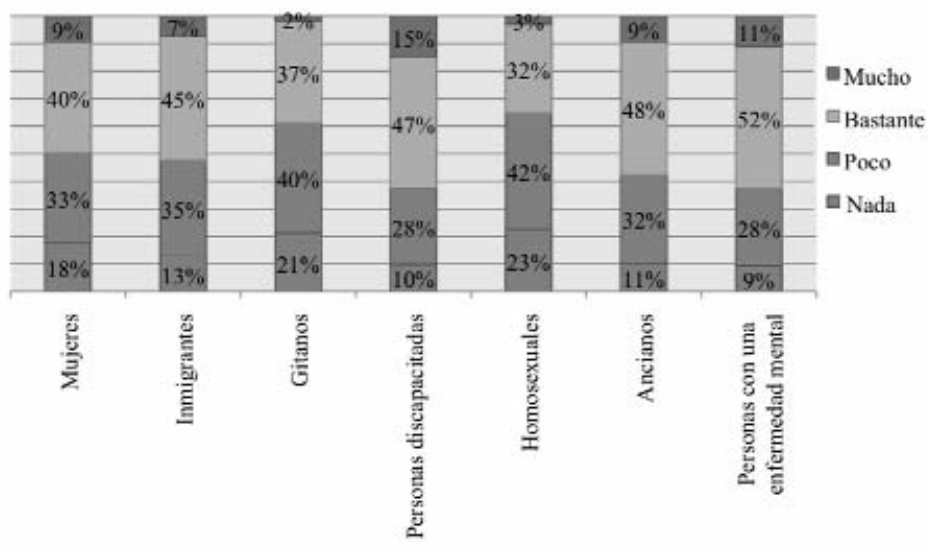

tres casos, sumado el

mucho con el bastante, las cifras se sitúan en el 62,5, 62 y 57,5 por ciento, siendo los primeros los que más sobresalen ante la opción mucho (15\%), seguidos de aquellos que padecen alguna alteración mental (11\%). En el resto de propuestas, destacan, en sentido negativo, los homosexuales y los gitanos, quienes acumulan, respectivamente, el 65 y 61 por ciento de un poco o nada. Los inmigrantes y las mujeres obtienen resultados parejos, es decir, ni a favor ni en contra, aunque bien podría afirmarse que las menos protegidas son las féminas, que reciben la opinión contraria de un $51 \%$ de los encuestados, frente al $48 \%$ que recogen los inmigrantes.

Ese reparto, casi equilibrado, esconde una percepción: que "nunca hay la suficiente información positiva sobre actuaciones que realizan esos colectivos como para com- 
pensar lo negativo". Vistas así las cosas, parece que lo prudente es no otorgar favores ni establecer cuotas en beneficio de nadie; pero para otros profesionales consultados el error hay que atribuírselo a la excesiva "corrección política" y a esa obsesión por "tratar de evitar lenguajes peyorativos" que, sobre todo en los últimos años, se han implantado en todos los medios, "y que ha llevado a dotar a las palabras de una carga significativa que en ocasiones no poseen. Si uno es negro, es negro; si es disminuido lo es. Eso no es más que una realidad; no es discriminar a nadie. Estamos en un punto en el que cualquier día no se va a poder decir nada" -apostillan-.

Cada pensamiento y postura encierran experiencias y una línea de trabajo que busca satisfacer a una sociedad fragmentada y sectorizada. Quizá esa realidad sea la justificación de que en Euskadi la suma del bastante y mucho del total de ítems, dividido por el números de éstos (siete), resulte una media del 51,2\%, lo que significa que, globalmente, todos los colectivos, a priori, podrían verse beneficiados en las informaciones.

En las entrevistas en profundidad realizadas a los profesionales de los medios andaluces, al plantearles si los grupos que histórica o tradicionalmente han sido desfavorecidos, o han estado en condiciones de "invisibilización", deberían de alguna forma ser compensados, se responde mayoritariamente que no especialmente, ya que "sería un gravísimo error", y que lo que procede es "escucharlos y no hacerlos invisibles, pero no hacer compensaciones". Reconocen saber que en ciertos documentos internos de los medios, como en el caso de RTVA, se establecen recomendaciones a los periodistas para tratar de otorgar un plus de positivismo en las in formaciones cuyos protagonistas son miembros de estos colectivos. Aun considerando que en ningún caso deben ser discrimi-

Gráfica 2.- Grado de apoyo de los periodistas a los grupos desfavorecidos

\section{Andalucía}

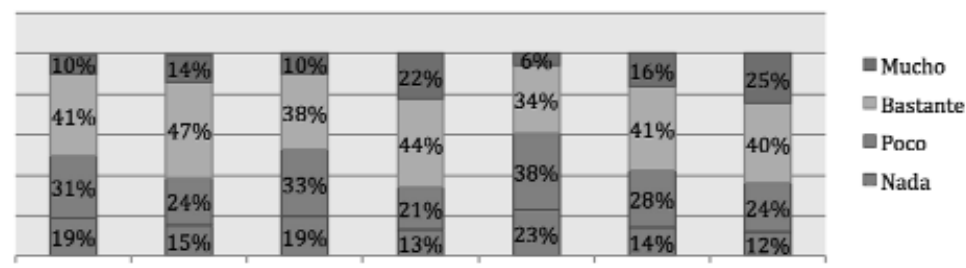
nados negativamente, mencionan también la dificultad de materializar dicha recomendación en su favor: en la práctica cotidiana se les confiere el protagonismo de las informaciones cuando realmente se trata de noticias, no en cualquier contexto que incluso no lleve aparejado el interés informativo.

En Andalucía, hasta un 55,1\% de los informadores encuestados se ha mostrado muy o bastante receptivo a ofrecer un trato favorable a los siete colectivos mencionados. En este sentido, es la comunidad donde este grado de sensibilización es mayor, muy lejos, por ejemplo, del 43,6\% de los periodistas catalanes.

No obstante, los resultados cuantitativos de los cuestionarios realizados reflejan 
que el colectivo que más adhesiones suscita es el de las personas discapacitadas (un $22,4 \%$ muy favorable y un $43,5 \%$ bastante), seguido de cerca por el de las personas con enfermedad mental (24,7\% y $39,5 \%)$. En el polo opuesto, los homosexuales destacan en último lugar, ya que sólo consiguen el $39,4 \%$ de las respuestas positivas $(5,8 \%$ y $33,6 \%)$, muy lejos del siguiente, los gitanos, que obtienen un $9,9 \%$ y $37,7 \%$, respectivamente.

En Cataluña, los profesionales de los medios de comunicación se muestran divididos ante la pregunta planteada. Hay quien piensa abiertamente que los mass media deben compensar a algunos grupos. Entienden que es "una forma de reparar una injusticia social histórica" o la manera de "implicarse socialmente", porque los medios "no pueden ser neutrales", en tanto que su labor tiene "una parte de denuncia social". La compensación a la que aluden se traduce en mayor presencia y visibilidad, al tiempo que en un trato e importancia más positivos y superiores. Esta posición la defienden, claramente, las mujeres.

Quien no comparte ese parecer arguye que toda discriminación, aunque sea positiva, es contraria a la igualdad y objetividad que los periodistas tienen la obligación de defender y procurar. En consecuencia, un trato favorable es calificado de injusto porque, además, las empresas de comunicación "no son ONGs". Estas tesis las mantienen mayoritariamente los hombres.

De entre todas las respuestas, la que más consenso logra es la de los discapacitados, tanto físicos como psíquicos, con una media del 57\%. Es el único caso en el que las respuestas favorables a un tratamiento informativo especial son superiores a las negativas.

Las opiniones se encuentran divididas, no obstante, ante las personas mayores: la mitad opta por apoyar la propuesta, mientras que el otro $50 \%$ se pronuncia en contra. Frente al resto de colectivos, la tendencia mayoritaria es contraria a que desde los medios se les preste una atención especial. Es comprensible, por tanto, que la media de las respuestas para las distintas propuestas sea del $43,6 \%$, lo que demuestra el escaso impulso y beneficio que se les quiere otorgar, salvo la ano-

Gráfica 3.- Grado de apoyo de los periodistas a los grupos desfa-
vorecidos

\section{Cataluña}

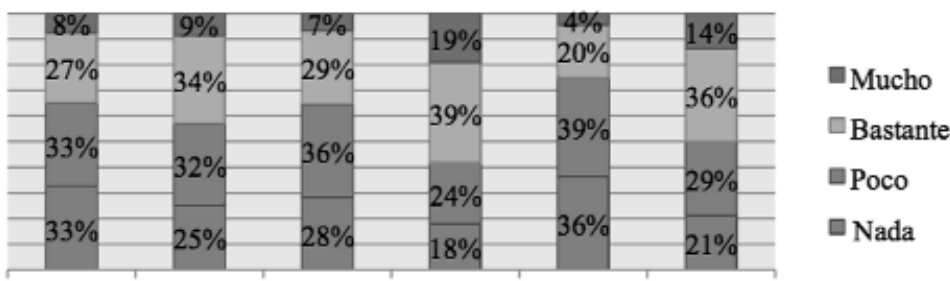
tada excepción.

Las entrevistas realizadas a los profesionales de la Comunidad de Madrid han mostrado que, en su práctica totalidad, están sensibilizados en alguna medida en relación 
Gráfica 4.- Grado de apoyo de los periodistas a los grupos desfavorecidos

\section{Madrid}

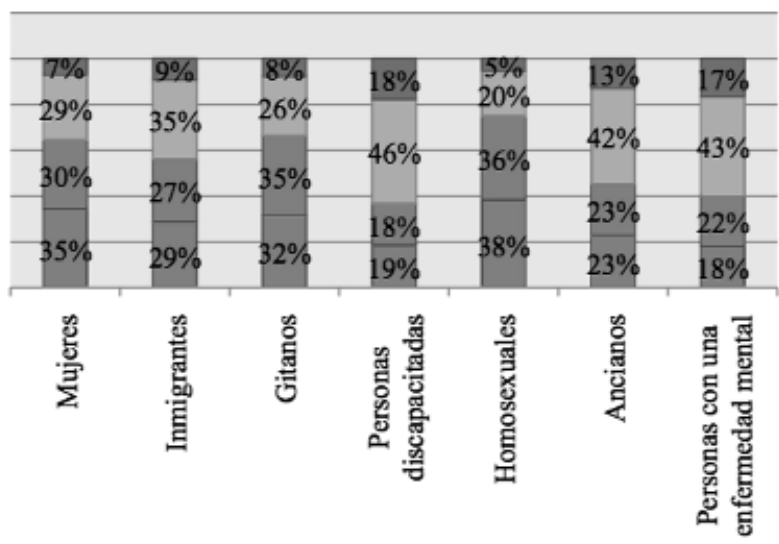

a los colectivos desfavorecidos. Sus manifestaciones en este sentido no concuerdan con los resultados obtenidos en los cuestionarios (que se analizan más adelante), según los cuales un $45,3 \%$ se manifiesta muy o bastante a favor de ayudarles, y una mayoría de $54,7 \%$ en contra.

Entre los que creen que no se les

debe compensar en su representación mediática, la gran idea que subyace es que ésa no es la misión de los medios, sino la de otras entidades. Cuatro de los entrevistados en profundidad afirman no creer en la discriminación positiva, sino que la labor del profesional es dedicarse a ofrecer información veraz. Para otros cuatro esta información veraz debe ser, además, igualitaria, sin discriminar positiva o negativamente, dando la misma relevancia a todos. Desde su punto de vista, favorecer especialmente a estos grupos podría perpetuar su discriminación, crear guetos y fomentar la xenofobia, por lo que la opinión pública debería percibirles como personas normales, y no estigmatizarlos ad eternum como desfavorecidos.

Por su parte, entre los que se muestran partidarios de compensarlos, consideran que educar en la igualdad, transmitir valores sociales, denunciar la injusticia y dar voz a los menos favorecidos, son objetivos, todos ellos, que forman parte del cometido y la responsabilidad de los profesionales del periodismo. En consecuencia, el tratamiento de la información debería favorecer la integración, mejorar su imagen pública y sensibilizar a la población hacia estas personas.

$\mathrm{Al}$ analizar las respuestas de los cuestionarios de los periodistas madrileños, puede observarse que se sienten más proclives a otorgar un tratamiento informativo especial a las personas con alguna discapacidad (17,8\% mucho y $45,6 \%$ bastante), a los que padecen alguna enfermedad mental $(16,3 \%$ y $43,3 \%)$ y a los ancianos $(13,2 \%$ y $41,6 \%)$. Por su parte, se muestran más reacios con los homosexuales $(36,4 \%$ poco y $38,4 \%$ nada), los gitanos $(34,6 \%$ y $31,6 \%)$ y las mujeres $(29,8 \%$ y $34,6 \%)$.

\section{Análisis por colectivos}

Los datos hasta aquí contemplados pretenden ser una aportación a los investigadores y a cuantos individuos o grupos, intervengan o no en el proceso de producción periodística, tengan interés por conocer la opinión de los informadores. Esa intención de contribuir anima a añadir, a partir de aquí, cada uno de los ítems, vinculados a Eus- 
kadi, Andalucía, Cataluña y Madrid, conjuntamente. Así, de una forma clara y directa, el análisis permite ver qué grado de apoyo o rechazo, en cuanto a recibir un trato favorable informativamente hablando, despiertan las mujeres, los inmigrantes, los gitanos y los ancianos, así como las personas con algún tipo de discapacidad, los homosexuales y quienes padecen una enfermedad mental. Ese mayor acercamiento al dato certero y puntual ayuda a saber, igualmente, las convergencias y divergencias que los mismos suscitan en las aludidas cuatro comunidades.

\subsection{Mujeres}

Respecto a las mujeres, como grupo históricamente desfavorecido, los periodistas encuestados en las cuatro comunidades se muestran mayoritariamente -en un $57,5 \%$ del total- poco o nada dispuestos a que reciban un tratamiento especialmente favorable, mientras que para un $42,5 \%$ sí debería considerarse.

Los más partidarios son los periodistas andaluces $(9,9 \%$ y $40,5 \%$ muy y bastante a favor, respectivamente), seguidos por los vascos $(9,4 \%$ y $40 \%)$, mientras que los que más manifiestan su posición negativa son los catalanes $(32,9 \%$ y $32,5 \%$ poco o nada favorables, respectivamente), y en una menor escala los madrileños (29,8\% y 34,6\%).

En función de su edad, se observa que en la Comunidad Autónoma del País Vasco los más inclinados a beneficiar a las mujeres son los profesionales de 41 a 50 años, con un $53,8 \%$ que se manifiestas muy o bastante partidarios. Por el contrario, los más opuestos son los ubicados entre los 31 y 40 años de edad. Los andaluces más jóvenes (20-39 años) están más conformes $(42,8 \%)$ que los que tienen entre 40 y 71 años $(36,4 \%)$.

En Cataluña, a medida que avanzan en edad (33,6\% en los jóvenes, $34,6 \%$ en las edades intermedias, y $36,1 \%$ en las avanzadas) aparecen más acordes al tratamiento informativo especial, aunque esta posición es siempre minoritaria. En realidad, los no partidarios ganan a los favorables en una proporción de dos a uno, que se repite con pocas variaciones en cada uno de los tramos.

En la Comunidad de Madrid los más reacios (poco o nada) a favorecer a las mujeres en la información son los profesionales jóvenes (menores de 30 años), con un $73,3 \%$, mientras que los que se muestran más de acuerdo son los mayores de 50 años $(39,9 \%)$.

Al indagar sobre el colectivo de las mujeres, lógicamente, adquiere un especial interés el cruce de los datos en función del género masculino o femenino de los profesionales encuestados. A priori podría pensarse que ellas se mostrarían partidarias en mayor proporción que ellos a ofrecer un trato especial de favor a las mujeres en las informaciones. Esa hipótesis se ha confirmado en todas las comunidades, aunque en dos de ellas por muy estrecho margen.

Así, en Euskadi las periodistas han sido más favorables que sus compañeros a promover a su mismo género, aunque sólo en una diferencia de dos puntos $(50 \%$ frente al 48\%). Por su lado, en Andalucía un 55,9\% de las informadoras son muy o bastante partidarias de que se les dé un tratamiento informativo especial, frente al $47,1 \%$ de los hombres. En Cataluña, un 48,3\% de las profesionales se han manifestado muy o bastante proclives, frente a un $37,4 \%$ de ellos. Finalmente, en Madrid es donde el por- 
centaje es menor. Solamente un $36 \%$ de las encuestadas se ha manifestado a favor, frente al $35,7 \%$ de los varones, quedando los dos sexos prácticamente a la par.

Otro factor que se ha considerado interesante a la hora de cruzar los datos obtenidos es el de la posición política de los entrevistados (izquierda, centro o derecha). En Euskadi las diferencias observadas en este sentido no son muy significativas, todas cercanas al 50\%, donde los extremos se ubican entre el 53,1\% favorable a las mujeres de los que se dicen de izquierdas y el 50,4\% de los que se consideran de centro. En Andalucía, aparecen mayores distancias, sobre todo entre los que se declaran de izquierdas $(53,8 \%)$ y los que manifiestan de derechas $(29,1 \%)$. La comunidad donde menor apoyo se encuentra es en Cataluña, donde todas las tendencias son en

Gráfica 5.- Comparativa por comunidades: Grado de apoyo de los periodistas a las mujeres.

\section{MUJERES}

mayor medida contrarias, sobre todo los de derecha $(84,8 \%)$, seguidos por los de centro $(76,3 \%)$ y los de izquierda $(62,3 \%)$. En la Comunidad de Madrid, los de izquierda son casi el doble de partidarios que los de derecha $(43,3 \%$ frente al $22,6 \%)$.

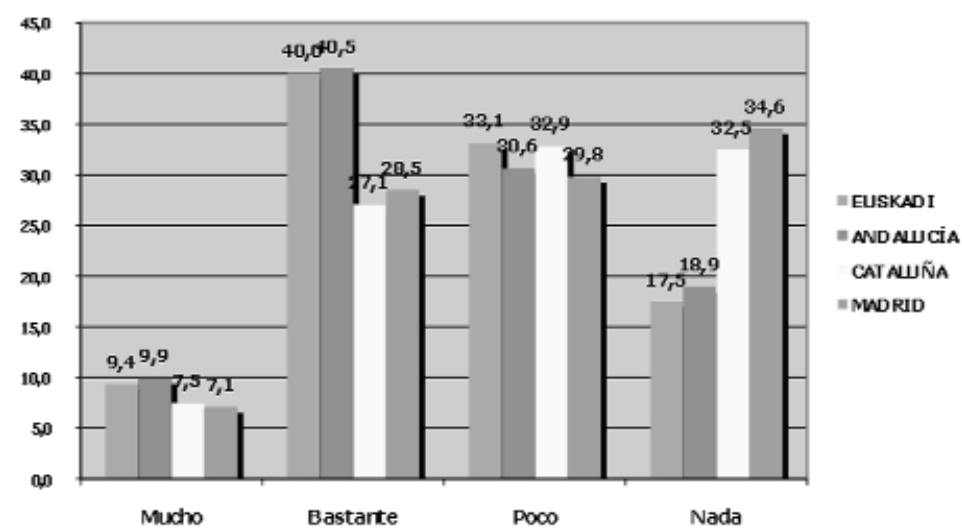

\subsection{Inmigrantes}

En el global de las cuatro Comunidades donde se ha realizado el estudio, la consideración del trato favorable o no a las personas inmigrantes es la que ha ofrecido unos resultados más parejos, puesto que un $50,1 \%$ del total de entrevistados se ha manifestado muy o bastante de acuerdo, frente al $49,9 \%$ posicionado como poco o nada partidario.

Si se entra a analizar los datos por comunidades, sí se observan diferencias entre ellas. En Andalucía un 14,4\% de los periodistas es muy favorable y un $46,6 \%$ bastante, por lo que se confiere como la más conforme de las cuatro. En segundo lugar, aunque algo alejada, aparece Euskadi, donde el $7 \%$ se muestra muy favorable y un $45,4 \%$ bastante.

Los comunicadores menos comprensivos respecto a su tratamiento especial son los catalanes, donde un $31,8 \%$ se manifiesta poco partidario y un $25,3 \%$ nada, seguidos de los madrileños, con un $27,1 \%$ y un $28,7 \%$, respectivamente, en sentido negativo.

En el cruce de datos en función de la edad de los entrevistados, puede observarse que en Euskadi los más favorables (mucho o bastante) lo conforma el grupo de edad entre los 41 y los 50 años $(60,3 \%)$ y que los menos partidarios son los de más de 50 años, con un 44,4\%. En Andalucía, en cambio, las respuestas positivas se obtienen de 
los de mayor edad (de 40 a 71 años), con hasta un 64,1\% de respuestas en este sentido, frente al 59,3\% de los de menor. Aunque en inferior proporción, en Cataluña también han sido los mayores los más propensos a favorecer a los inmigrantes, con un $47,4 \%$ de periodistas que tienen o superan los 56 años, frente al $41,5 \%$ de los comprendidos entre 36 y 55 años. En Madrid se reitera la tendencia con el grupo de más de 50 años $(48,9 \%)$. En el lado opuesto están los más jóvenes (menores de 30 años), que sólo lo admiten en un $37,5 \%$.

Al realizar el cruce según el género de los entrevistados, en Euskadi y en Madrid los profesionales varones están muy o bastante posicionados a tratar

Gráfica 6.- Comparativa por comunidades: Grado de apoyo de los periodistas a los inmigrantes

INMIGRANTES

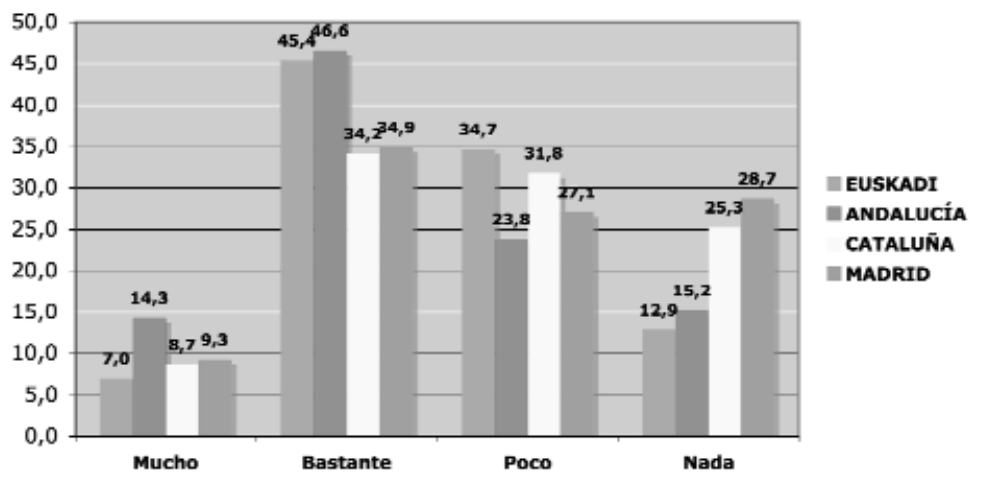

de modo positivo a los inmigrantes, en un $56 \%$ y un $45,7 \%$ respectivamente, frente a las $48,9 \%$ y $41,6 \%$ de las periodistas. En Andalucía los resultados se invierten: las mujeres son más partidarias que los varones $(66,7 \%$ frente al $57,5 \%)$.

En las cuatro comunidades se aprecian resultados significativos en cuanto al posicionamiento ideológico de los entrevistados y su actitud ante los inmigrantes. En todas ellas, con una excepción en Andalucía, a medida que se es más de izquierdas más inclinado se está a su tratamiento especial. De ese modo, en Euskadi, hasta un 65,6\% el mayor de todos los porcentajes- de los que sitúan en el campo de la izquierda es favorable a su consideración especial, frente a un $50 \%$ de los de derecha. En la comunidad andaluza, el $64,7 \%$ de los de izquierda, el $40 \%$ de los de centro y el $42,3 \%$ los de derecha también lo están. No obstante, aquí aparece un dato contradictorio: el $71,4 \%$ de los que dicen ser muy de derechas declara estar bastante de acuerdo. En Cataluña, la variable ideológica se muestra como la más determinante: entre los declarados de izquierda un $47,5 \%$ son favorables, un $25,5 \%$ de los de centro y un $23,9 \%$ de los de la derecha. En Madrid, el 51,3\% de los profesionales de izquierda dicen estar a favor, mientras que entre los de derecha, el $87,3 \%$ es contrario.

\subsection{Gitanos}

Según los datos totales obtenidos, el colectivo gitano es, tras el de los homosexuales, el que menor grado de apoyo recibe: hasta un $61,1 \%$ de todas las respuestas de todas las comunidades han sido bastante o muy contrarias a ese favoritismo.

Entre todas las zonas geográficas participantes en el estudio destaca el rechazo de Madrid (34,6\% de poco partidarios y un 31,6\% de nada partidarios), seguida de Ca- 
taluña $(36,2 \%$ y $28,3 \%)$ y País Vasco $(40,1 \%$ y $21,2 \%)$. Andalucía, por su parte, es la que en mayor medida lo acepta (37,7\% bastante y $9,9 \%$ mucho), aun siendo igualmente ésta la opción minoritaria.

Respecto a la edad de los encuestados en Euskadi se observa que, según aumentan los años, el grado de aceptación asciende. El porcentaje va aumentando progresivamente desde los menores de 30 años, que sólo lo aprueban en un $34,8 \%$, hasta los mayores de 50 que lo hacen en un 44,4\%. En Andalucía, de modo similar, son los que tienen de 40 a 71 años quines, sobre todo, se muestran partidarios $(55,2 \%)$, frente al 43,5\% de los más jóvenes (20-39 años). Igualmente, en Cataluña los que superan los 56 años son los más comprensivos, con un $43,2 \%$, situándose la siguiente franja de edad (36-55 años) en el 33,9\%. En Madrid vuelve a repetirse la tendencia: los más jóvenes (menores de 30 años) son los mayormente contrarios a ningún tipo de favor $(76,4 \%)$, mientras que los mayores de 50 lo son en menor medida $(59,1 \%)$.

En el cruce de datos según el género de los periodistas, en Euskadi, sólo el 34,7\% de ellas estiman algún tipo de favor hacia los gitanos, frente al $43,5 \%$ de ellos. En Andalucía se invierte la tendencia, al reflejar que un 51,2\% de las mujeres son en alguna medida favorables, frente al 45,4\% de los hombres. En Madrid, los resultados están equilibrados: un 33,6\% de féminas y un 34\% de varones se muestran partidarios.

En cuanto a la ideología, vuelve a manifestarse la tendencia apreciada en los anteriores colectivos: cuanto más a la derecha, mayor es la falta de apoyo. En el País Vasco los de izquierda favorables a los gitanos se aproximan a la mitad $(48,4 \%)$, los de centro se quedan en el $38,1 \%$, mientras que los de derecha son sólo partidarios en un 10\%. En Andalucía los profesionales de derechas sólo aceptan un trato especial en

Gráfica 7.- Comparativa por comunidades: Grado de apoyo de los periodistas a los gitanos

\section{GITANOS}

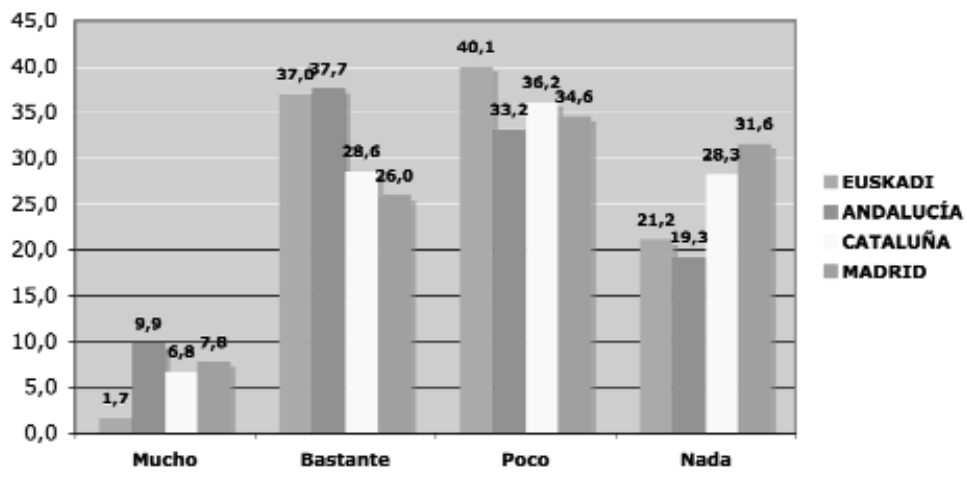

un $29,1 \%$ y los de izquierda en un $51,6 \%$. En Cataluña el $39,7 \%$ de los que se declaran de izquierda lo consideran positivo, mientras que entre los de derecha, sólo el $17,4 \%$ lo hace. En la comunidad madrileña, el $42,9 \%$ de los de izquierda y el $15 \%$ de los derecha lo acep$\tan$.

\subsection{Discapacitados}

Respecto a los colectivos analizados, los resultados globales de las cuatro comunidades reflejan que, de todos ellos, el que ha suscitado más respuestas afirmativas hacia su consideración especial, es el de las personas con algún tipo de discapacidad, con- 
cretamente, hasta un $62,5 \%$ de todas las respuestas han sido muy o bastante favorables. En mayor medida lo han hecho en Andalucía, donde hasta un $43,5 \%$ se ha manifestado bastante a favor y un $22,4 \%$ muy a favor, seguida de Madrid (45,6\% y $17,8 \%$ ), Euskadi (47,4\% y 15,1\%) y, por último, Cataluña $(39,2 \%$ y $19,1 \%)$.

Teniendo en cuenta la variable de la edad de los encuestados, en el País Vasco son los profesionales situados entre los 31 y los 40 años $(72,2 \%)$ los más dispuestos a potenciar a las personas discapacitadas. Los menores de 30 años lo hacen en un $57 \%$. Cifras cercanas se mantienen Andalucía donde también destacan los que tienen de 40 a 71 años $(71,8 \%)$ mientras que los más jóvenes, de 20 a 39 años, lo son en un $62,8 \%$. De igual manera, en la Comunidad de Madrid son los de más de edad (mayores de 50) los que se posicionan a favor de un trato especialmente generoso $(69,1 \%)$, en tanto que los más jóvenes (menores de 30 años) lo hacen considerablemente en menor grado (50\%).

La variable del sexo de los profesionales indica que en Euskadi el 67,3\% de los hombres y el $58 \%$ de las mujeres encuestadas son partidarios, en mayor o menor medida, del tratamiento especial del colectivo. En la Comunidad andaluza las cosas están más equidistantes, aunque se invierte la mayoría: el 69\% de las mujeres y el 64\% de los hombres contestan positivamente. En Madrid nuevamente son los varones mayoría, con un $66,3 \%$, mientras que ellas aceptan algún trato de favor en un 59\% de las respuestas.

Al observar el posicionamiento ideológico de los entrevistados, se aprecia que en el País Vasco un elevado 80\% de los entrevistados (el mayor de todos los registros) que se declaran de derechas son partidarios del trato especial favorable para con las personas discapacitadas. Este porcentaje baja hasta el $64,1 \%$ en el caso de los profesionales de izquierda y de centro. En Andalucía, en cambio, son los de izquierda $(67,1 \%)$ los que superan a los de derecha $(58,2 \%)$. En Cataluña no se observan diferencias significativas en este aspecto, y en Madrid, los de izquierda $(65,2 \%)$ duplican el porcentaje favorable de los de dereGráfica 8.- Comparativa por comunidades: Grado de apoyo de los periodistas a las personas discapacitadas

PERSONAS DISCAPACITADAS

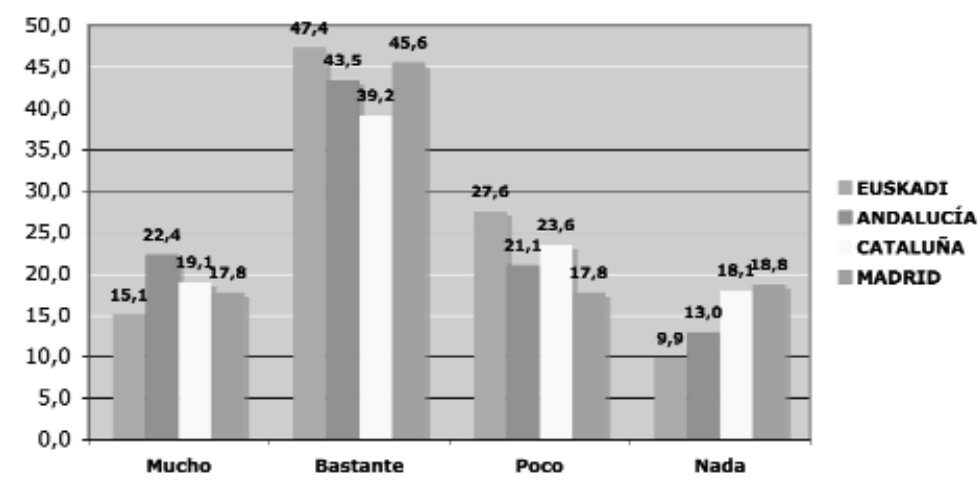
cha $(31,9 \%)$.

\subsection{Homosexuales}

Los homosexuales han sido los que menor grado de aceptación -y, por ende, mayor rechazo- ha suscitado entre todas las alternativas presentadas. Sólo un $31,2 \%$ de todos los profesionales encuestados lo ha considerado oportuno. Si se pone en relación con 
el anterior colectivo de personas discapacitadas, puede observarse que prácticamente se reduce a la mitad el nivel de aceptación.

Como puede apreciarse en la gráfica 9 , los más reacios son los catalanes y los madrileños: en Madrid, un 38,4\% se pronuncia totalmente en desacuerdo, mientras que el 38,7\% está bastante en contra; en Cataluña un 36,3\% está muy en contra y un 38,7\% bastante. En ambas comunidades, sólo uno de cada cuatro entrevistados cree correcto tratar favorablemente a los homosexuales.

Aunque la mayoría sigue siendo negativa, en Andalucía y Euskadi se amortiguan las discrepancias, llegando a estar en la primera de esas comunidades cerca del $40 \%$ a favor en alguna medida, y en la comunidad vasca en un $35,3 \%$.

Gráfica 9.- Comparativa por comunidades: Grado de apoyo de los periodistas a los homosexuales

Al cruzar los datos con la edad de los encuestados, se aprecia que los profesionales mayores de 50 años y los comprendidos entre 31 y 40 (41,7\% y $40,3 \%$, respectivamente) aventajan en alrededor de ocho puntos a los otros dos

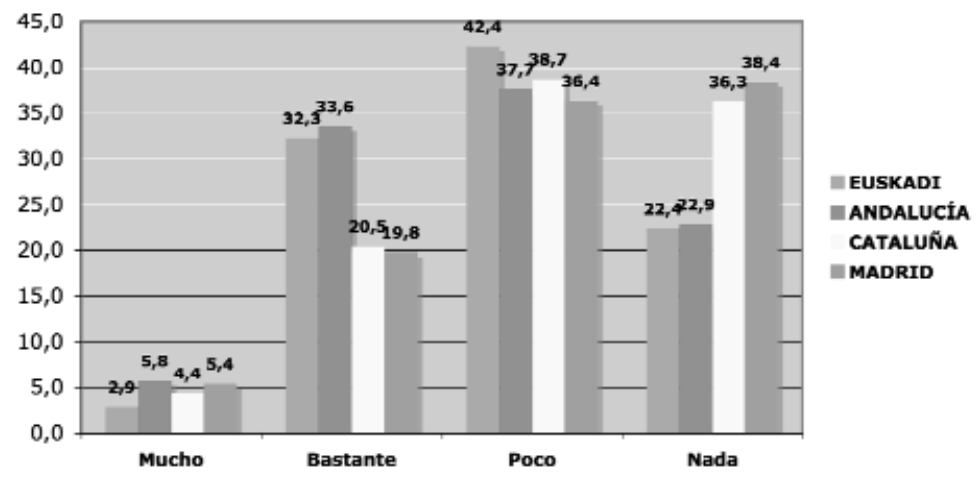

grupos de edad, a los menores de 30 años $(33,5 \%)$ y a los de entre 41 y 50 años $(32,1 \%)$. En Andalucía, los jóvenes de 20 a 39 años son los que más lo apoyan, con un $40,7 \%$ a favor, en tanto que los mayores (de 40 a 71 años), lo hacen en un 37,2\%. En la Comunidad de Madrid, los que superan los 50 años son los más partidarios $(29,5 \%)$ y los menores de 30 años, los menos $(19,6 \%)$. En Cataluña se observa que, de forma similar a los casos de mujeres e inmigrantes, es el segmento de más edad el que reúne a un número superior de partidarios del trato especial (con un 30,2\% del total). Los que se encuentran en edades intermedias y los jóvenes continúan situándose muy cerca uno del otro ( $24,5 \%$ y $23,7 \%$, respectivamente).

En cuanto al factor ideológico, en Euskadi los de izquierda se manifiestan mucho más claramente favorables $(42,2 \%)$ que los de derecha (10\%), mientras que los de centro lo son en un 35,9\%. En Andalucía se repite la tendencia: un 46,4\% de los de izquierda (el dato más favorable de todos) y un $9,5 \%$ de los de derecha lo admiten. También se confirma en Cataluña, aunque en menor medida, ya que un 27,9\% de los de izquierda se dicen partidarios, frente a los 14,7 de centro y un $8,7 \%$ de los de derecha. En Madrid, finalmente, es donde se aprecia un mayor rechazo, ya que solamente el $34 \%$ de los de izquierda y un $9 \%$ de los de derecha declaran tomarlo en consideración. 


\subsection{Ancianos}

De entre todas las comunidades, Euskadi es la que se muestra bastante dispuesta a proteger a sus ancianos. Si ese bastante se suma al mucho, los resultados se equilibran y puede decirse que los periodistas de las cuatro zonas comparten, prácticamente, la misma opción, siendo la media resultante del $54,9 \%$. Individualmente, Andalucía y Euskadi se sitúan al mismo nivel de protección (57,6\%). Le sigue Madrid, con un $54,8 \%$. La disposición es menor en Cataluña, donde la cifra se sitúa en el 49,8\%, dándose un empate casi técnico entre partidarios y detractores.

En esa misma zona, las diferencias se diluyen incluso entre grupos de edades, salvo con los más jóvenes que se sitúan en el 45,5\%. Algo similar ocurre entre las diferentes afinidades políticas. Si bien, los de centro reclaman en mayor medida un tratamiento especial: $20 \%$, frente al $12,7 \%$ del segmento de la izquierda y el $4,4 \%$ de la derecha.

La variable edad vuelve a surgir como elemento diferenciador entre los profesionales madrileños y andaluces. Dentro de los primeros, son más benévolos los que superan la cincuentena $(62,7 \%)$. Lo son en menor medida los que tienen menos de 30 (34\%). En Andalucía, por su parte, las diferencias entre unos y otros son escasas: los que están en la franja de 40 a 71 años acumulan el $61,1 \%$ y los que tiene por debajo de 39 suman 55,9\%. Tampoco presentan excesivas variaciones los encuestados en Euskadi. En las franjas que acogen a los que tienen de 31 a 40 años, de 41 a 50 y de 50 en adelante, las diferencias son estrechas: 71,64 y 68 por ciento.

El ser anciano acerca a hombres y mujeres madrileños, aunque se mantienen algunas distancias. Si para el género masculino su presencia en los medios podría recibir algún trato de favor (56\%), para el femenino la cifra se sitúa en el $52,7 \%$. La disonancia reaparece ante posiciones políticas, mostrando mejor disposición (bastante y mucho) los que se consideran de izquierdas $58,8 \%$, y poco o nada los de derechas $(52,7 \%)$.

Igual tónica resalta en Euskadi, prevaleciendo los hombres frente a las mujeres: $64 \%$ frente a $53 \%$. No pasa lo mismo cuando se introduce la ideología como elemento

Gráfica 10. Comparativa por comunidades: Grado de apoyo de los periodistas a los ancianos

\section{ANCIANOS}

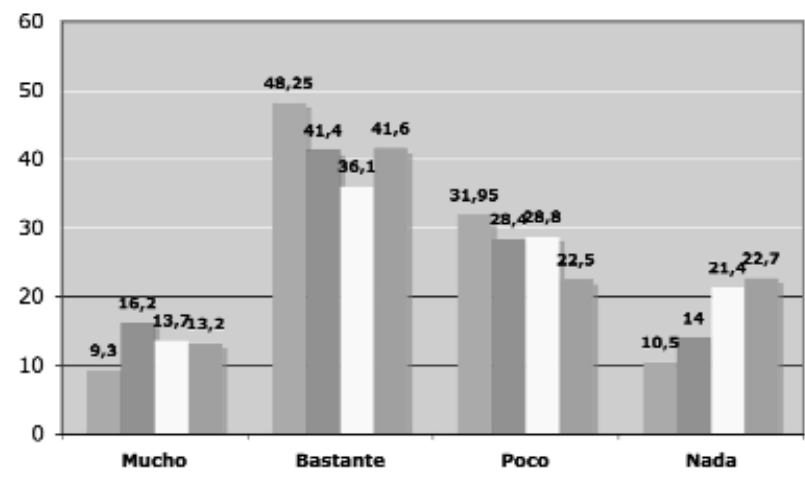

a comparar, ya que en el caso del País Vasco no existen diferencias significativas.

Los datos de los andaluces invierten las respuestas de los anteriores, al ser los varones menos partidarios que sus compañeras de establecer cuotas de mejora o beneficio: $55,1 \%$ frente a 
$61,9 \%$. No hay cambios si se comparan las posturas de los que se manifiestan de izquierdas o de derechas, prevaleciendo la opción desfavorable de éstos últimos frente a la de los anteriores.

\subsection{Personas con enfermedad mental}

Cuando los profesionales de los medios tienen que posicionarse ante la cuestión de si favorecerían en sus informaciones a personas con enfermedad mental, el sí se sitúa en el 61,6\% de media entre los resultados alcanzados en Andalucía, País Vasco, Madrid y Cataluña. El mayor porcentaje lo aporta la primera de las comunidades, donde el $64,2 \%$ de los periodistas comparten esa respuesta. A corta distancia lo hacen los informadores de Euskadi (62,5\%), seguidos de los madrileños y de los catalanes, con porcentajes del $59,9 \%$ y $59,4 \%$, respectivamente.

En Madrid, la unanimidad de las voces favorables salen de los de más edad (ma-

Gráfica 11.- Comparativa por comunidades: Grado de apoyo de los periodistas a las personas con enfermedad mental

PERSONAS CON ENFERMEDAD MENTAL

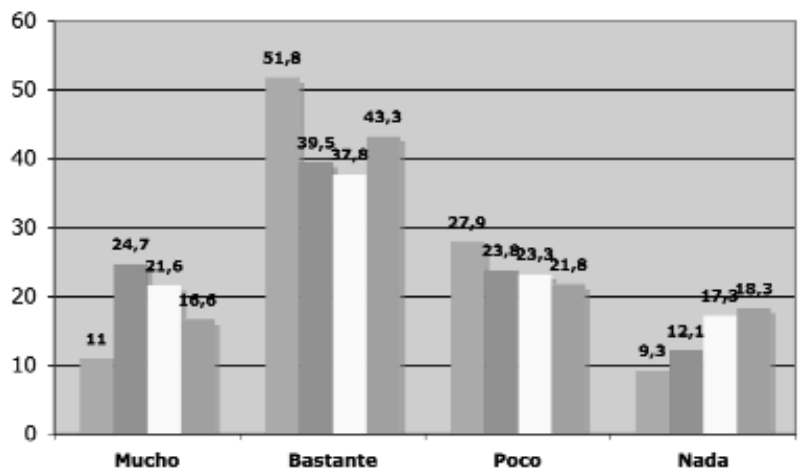

yores de 50 años). Dentro de ese grupo, el mucho y el bastante llega a $65,4 \%$, unas décimas por encima de los catalanes que superan los 55 años $(62,4 \%)$. En ambos territorios los

EUSKADI ANDALUCIA MADRID jóvenes por debajo de los 30 años y entre 30 y 50 marcan discrepancias notorias $(48,2 \%$ y $54,9 \%$, respectivamente).

Andalucía y Eus-

kadi presentan sus particularidades. En la primera, las diferencias apenas se dejan sentir: $68,3 \%$ y $61,8 \%$ establecen las tendencias de los mayores y los más jóvenes. En la segunda, son los que tienen entre 31 y 40 años los que las imprimen, aunque con escaso margen con los que llegan a la cincuentena, dado que se reparten el 71 y el 68 por ciento.

Cuando el género se suma a la variable aquí analizada son los comunicadores madrileños y vascos los que se distancian de sus compañeras, al ser más partidarios de proporcionar un trato diferenciado $(63,7 / 54,7 \%$ y $66 / 59 \%$, respectivamente). Esa tendencia se aproxima e invierte en Andalucía, donde las mujeres representan el 67,2\% de las respuestas mucho y bastante, y los hombres el $62,1 \%$.

La ideología apenas si denota posturas opuestas en las áreas geográficas del estudio. Tal es así que los datos son irrelevantes en Cataluña, casi paritarios en Madrid (derecha $61,1 \%$, izquierda $60,8 \%$ y centro $58 \%$ ) y con una ligera inclinación por parte de la derecha vasca y andaluza hacia actitudes poco favorables. En el País Vasco esa opción alcanza cotas del 70\%, posicionándose los de izquierda en el $68,8 \%$ y los de centro en un $64,6 \%$. 


\section{Conclusiones}

- En los códigos europeos la raza, la religión, el origen nacional y el color son aspectos a proteger. Se deduce, en consecuencia, que el cuidado en el tratamiento informativo de los inmigrantes es una preocupación fundamental. Las mujeres y los mayores también son objeto de protección.

- Los periodistas de Euskadi, Andalucía, Cataluña y Madrid son mayoritariamente poco o nada partidarios de primar en las informaciones a los colectivos desfavorecidos, si bien, la diferencia con los que dicen sentirse muy o bastante favorables es muy escasa: 51,2 y 48,8 por ciento, respectivamente.

- Andalucía es la comunidad que muestra una mayor predisposición, especialmente con las personas discapacitadas o enfermas mentales, así como con los inmigrantes, los ancianos y las mujeres. Euskadi comparte ese orden, exceptuando a las mujeres.

- Cataluña únicamente es proclive a quienes padecen alguna enfermedad mental y a los discapacitados. Madrid contempla esas dos mismas propuestas, además de la de los ancianos.

- Los gitanos y los homosexuales no cuentan con el apoyo de los informadores de ninguno de los cuatro territorios. Las mujeres sólo encuentran respuestas superiores al 50\% en Andalucía.

- La edad es un factor que favorece a los colectivos en general. Salvo en Andalucía, donde los más jóvenes otorgan mayor apoyo a las mujeres, en el resto de autonomías son los que superan los 40 años quienes adoptan posturas más laxas.

- El género no es una variable diferenciadora, aunque sí para el colectivo de las mujeres y el de los ancianos, que son mas protegidos por las féminas.

- Los informadores que se declaran de izquierda son los más favorables con el conjunto de los colectivos analizados. Destaca, sin embargo, el hecho de que en todas las comunidades el $90 \%$ de los encuestados que dicen ser de derechas se muestran contrarios a los homosexuales.

- Los grupos que más homogéneamente son aceptados por todas las tendencias políticas son los discapacitados.

\section{Referencias bibliográficas}

ALLIVERI, Stefano (2010): “¿El Islam en Europa se hace europeo?”, en Afkar/Ideas. Revista trimestral para el diálogo entre el Magreb, España y Europa, no 25. [Puede consultarse en: www-afkar-ideas.com].

AZNAR, Hugo (1997): "El debate en torno a la utilidad de los códigos deontológicos del periodismo". En: Anàlisi, no 20, (pp. 125-144).

COMISIÓN EUROPEA (2007): Europe's demographic future: facts and figures on challenges and opportunities. [Puede consultarse en http://ec.europa.eu/employment_social/spsi/docs/social_situation/demo_report_2007_en.pdf

EUROSTAT (2009): Population of foreign citizens in EU27 in 2008. En Eurostat Newsrelease: http://epp.eurostat.ec.europa.eu/portal/page/portal/population/ publications/migration_asylum 
PÉREZ FUENTES, Juan Carlos (comp., 2004): Ética periodística. Principios, códigos deontológicos y normas complementarias. Bilbao, Universidad del País Vasco.

TUCHMAN, Gaye (1983): La producción de la noticia. Barcelona: Gustavo Gili.

\section{Códigos deontológicos consultados}

UNESCO: Principios Internacionales de ética profesional del periodismo (1983)

FEDERACIÓN INTERNACIONAL DE PERIODISTAS: Declaración de principios sobre la conducta de los periodistas (1986)

CONSEJO DE EUROPA: Resolución 1003 sobre la Ética del Periodismo (1993)

ALEMANIA: Consejo Alemán de la Prensa: Normas Fundamentales del Periodismo (2006)

AUSTRIA: Consejo de Prensa Austriaco: Código de Ética para la Prensa Austriaca (1999)

BÉLGICA: Asociación de Editores de Periódicos, Asociación General de Periodistas Profesionales de Bélgica y Federación Nacional de Semanarios de Información: Código de Principios Periodísticos (1982)

BULGARIA: Medios de Comunicación Búlgaros: Código Ético de los Medios de Comunicación Búlgaros (2004)

CHIPRE: Unión de Periodistas y Asociación de Editores de Diarios y Revistas y Propietarios de medios electrónicos: Código de Conducta de los Periodistas (1997)

DINAMARCA: Unión Nacional de Periodistas: Código Nacional de Conducta (1992)

ESLOVAQUIA: Sindicato Eslovaco de Periodistas: Código de Ética (1990)

ESLOVENIA: Asociación de Periodistas de Eslovenia: Código de Ética ( 2002)

ESPAÑA: Federación de Asociaciones de Periodistas de España (FAPE): Código Deontológico de la Profesión Periodística (1993)

ESTONIA: Consejo Estonio de la Prensa: Código de Ética para la Prensa de Estonia (1997)

FINLANDIA: Unión de Periodistas de Finlandia: Pautas para los periodistas (2005)

FRANCIA: Carta de los Deberes Profesionales de Periodistas franceses (1938)

GRECIA: Federación Panhelénica de Sindicatos de Periodistas: Código Ético para los Periodistas Profesionales (1998)

HUNGRÍA: Asociación Nacional de Periodistas Húngaros: Código Ético (2007)

IRLANDA: Sindicato Nacional de Periodistas: Código de Conducta (2007)

Consejo de Prensa de Irlanda: Código de Buenas Prácticas para Prensa y Publicaciones Periódicas (2008)

ITALIA: Federación Nacional de la Prensa Italiana y Consejo Nacional de Periodistas: Carta de los Deberes de los Periodistas (1993) 
LETONIA: Unión de Periodistas de Letonia: Código de Ética (1992)

LITUANIA: Código de Ética de los Periodistas y Editores de Lituania (2005)

LUXENBURGO: Consejo de Prensa: Código Deontológico (2004)

MALTA: Club de Prensa de Malta: Código de Ética Periodística (2000)

POLONIA: Asociación de Periodistas Polacos: Código de Ética Periodística (2001)

PORTUGAL: Unión de Periodistas: Código de Ética de los Periodistas (1993)

RUMANÍA: Convención de las Organizaciones de Medios: Código de Ética de la Prensa (2004)

REINO UNIDO: Unión de Periodistas: Código de Conducta (2007). Comisión de Reclamaciones a la Prensa: Código de Prácticas (2007)

REPÚBLICA CHECA: Sindicato de Periodistas: Código Ético de los Periodistas (1999)

SUECIA: Consejo de Prensa y Comisión Mixta de las Organizaciones de Medios de Comunicación: Código de Ética para la Prensa, la Radio y la Televisión (2006) 